OPEN ACCESS

Edited by: Shereen Nizari,

University College London,

United Kingdom

Reviewed by:

Michael S. Stringer,

University of Edinburgh,

United Kingdom

Rita Moretti,

University of Trieste, Italy

${ }^{*}$ Correspondence:

Aleksander Dębiec

adebiec@wim.mil.pl

Received: 19 June 2021 Accepted: 13 September 2021

Published: 20 October 2021

Citation:

Staszewski J, Dẹbiec A, Skrobowska E and Stẹpień A (2021) Cerebral Vasoreactivity Changes Over Time in Patients With Different Clinica Manifestations of Cerebral Small Vessel Disease.

Front. Aging Neurosci. 13:727832.

doi: 10.3389/fnagi.2021.727832

\section{Cerebral Vasoreactivity Changes Over Time in Patients With Different Clinical Manifestations of Cerebral Small Vessel Disease}

\author{
Jacek Staszewski ${ }^{1}$, Aleksander Dȩbiec ${ }^{1 *}$, Ewa Skrobowska ${ }^{2}$ and Adam Stępień ${ }^{1}$ \\ ${ }^{1}$ Military Institute of Medicine, Clinic of Neurology, Warsaw, Poland, ${ }^{2}$ Department of Radiology, Military Institute of Medicine, \\ Warsaw, Poland
}

Objectives: Endothelial dysfunction (ED) has been linked to the pathogenesis of cerebral small vessel disease (SVD). We aimed to assess ED and cerebrovascular reactivity (CVR) in the patients with a diverse manifestation of SVD, with similar and extensive white matter lesions (WMLs, modified Fazekas scale grade $\geq 2$ ), compared with a control group (CG) without the MRI markers of SVD, matched for age, gender, hypertension, diabetes, and to evaluate the change of CVR following 24 months.

Methods: We repeatedly measured the vasomotor reactivity reserve (VMRr) and breath-holding index $(\mathrm{BHI})$ of the middle cerebral artery $(\mathrm{MCA})$ by the transcranial Doppler ultrasound (TCD) techniques in 60 subjects above 60 years with a history of lacunar stroke (LS), vascular dementia (VaD), or parkinsonism (VaP) (20 in each group), and in 20 individuals from a CG.

Results: The mean age, frequency of the main vascular risk factors, and sex distribution were similar in the patients with the SVD groups and a CG. The VMRr and the BHI were more severely impaired at baseline (respectively, $56.7 \pm 18 \%$ and $0.82 \pm 0.39$ ) and at follow-up (respectively, $52.3 \pm 16.7 \%$ and $0.71 \pm 0.38$ ) in the patients with SVD regardless of the clinical manifestations (ANOVA, $p>0.1$ ) than in the CG (respectively, baseline $\operatorname{VMRr} 77.2 \pm 15.6 \%$, BHI $1.15 \pm 0.47, p<0.001$; follow-up $\operatorname{VMRr} 74.3 \pm$ $17.6 \%, \mathrm{BHI} 1.11 \pm 0.4, p<0.001)$. All the assessed CVR measures (VMRr and BHI) significantly decreased over time in the subjects with SVD (Wilcoxon's signed-rank test $p=0.01)$, but this was not observed in the CG $(p>0.1)$ and the decrease of CVR measures was not related to the SVD radiological progression $(p>0.1)$.

Conclusions: This study provided evidence that the change in CVR measures is detectable over a 24-month period in patients with different clinical manifestations of SVD. Compared with the patients in CG with similar atherothrombotic risk factors, all the CVR measures (BMRr and BHI) significantly declined over time in the subjects with SVD. The reduction in CVR was not related to the SVD radiological progression.

Keywords: neurovascular unit (NVU), cerebral small vessel disease, cerebrovascular reactivity (CVR), endothelial dysfunction, neurovascular coupling 


\section{BACKGROUND}

Small vessel disease (SVD) is one of the most important cerebral microangiopathy, responsible for the majority of lacunar stroke (LS), vascular dementia (VaD), and parkinsonism (VaP) cases (Pantoni, 2010). The basic mechanism of the cerebral vessels alterations in the SVD is linked to endothelial dysfunction (ED), but whether ED only reflects the load of atherothrombotic risk factors or if it is specific to SVD has not been clearly defined (Forsberg et al., 2018). The neurovascular unit (NVU) concept accentuates the symbiotic association between brain cells, cerebral blood vessels, and subsequently cerebral blood flow (CBF) (Muoio et al., 2014). The endothelium and vascular smooth muscle within the NVU forms the basis of blood-brain-barrier (BBB), and contribute to the neurovascular coupling that is the response of the cerebral vessel to the changes in neural activity (Attwell et al., 2010). The SVD is a dynamic and progressive pathology involving variable components of $\mathrm{NVU}$ and $\mathrm{BBB}$, however, the long-term clinical effects and outcomes usually differ between patients (Kisler et al., 2017). The recent studies have shown that the signaling pathways in the NVU control diverse processes, e.g., blood clotting and $\mathrm{CBF}$, nevertheless it is not known precisely how CBF dysregulation translate to the disorders associated with neurovascular dysfunction, such as SVD. Cerebrovascular reactivity (CVR) is a measure of the capability of adaptive changes to vasodilatory stimuli (e.g., change in $\mathrm{pCO}_{2}$ due to $\mathrm{CO}_{2}$ inhalation, voluntary apnea, hyperventilation, or acetazolamide), and reflects the compensation of the collateral flow, therefore it can be used to indirectly assess and monitor the sequences of cerebral ED and the progression of vascular disease (Lavi et al., 2006). The reduced CVR indicates the impairment of NVU and regulatory mechanisms of $\mathrm{CBF}$ which result in neurovascular uncoupling. There are no biological markers to accurately assess brain vasoreactivity; however, CVR can be evaluated by various tools, such as single photon emission computed tomography (SPECT), PET, various MRI techniques, and transcranial Doppler ultrasound (TCD) (Terborg et al., 2000). Currently, one of the most widely used methods to measure the CVR is MRI, and it offers advantages over the use of radiolabeled products while maintaining regional specificity (Sleight et al., 2021). Though no direct anatomical information can be obtained, TCD permits for the evaluation of mean flow velocity (MFV) changes in the major cerebral arteries after a vasodilative stimulus and it provides complementary information of the brain hemodynamics (Ringelstein et al., 1988; Ebrahim et al., 2019; Burley et al., 2021a). The results of the TCD examination (MFV and pulsatility index) and the ventilation tests correlate with those obtained by other methods, and the value of TCD in the evaluation of CVR impairment, e.g., in the asymptomatic or symptomatic individuals with the brain white matter lesions (WMLs) has been established (Maeda et al., 1993; Marcos et al., 1997; Ghorbani et al., 2015; Fu et al., 2019). The assessment of CVR can be achieved with the bilateral recording of MFV in the middle cerebral artery (MCA) with acceptable reproducibility and inter-rater reliability using carbogen inhalation (McDonnell et al., 2013). Recently, we have found that the cerebral vasodilator responses to breath hold and hyperventilation were abnormal in the patients with $\mathrm{VaD}$, LS, and VaP caused by SVD, and they were severely impaired when compared with the controls matched for the main vascular risk factors and free from the cerebrovascular events (Staszewski et al., 2019). Most of the studies assessing CVR in SVD did not evaluate the CVR changes over time or had unmatched control groups (CGs) (Thrippleton et al., 2018). Therefore, our study aimed to investigate the CVR changes over 24 months in the subjects with diverse manifestations of SVD (LS, VaD, and VaP), with similar and extensive radiological burdens of the disease and compare with a carefully selected CG without MRI markers of SVD, free of cerebrovascular events, and matched for major vascular comorbidities.

\section{MATERIALS AND METHODS}

\section{Participants}

We analyzed the patients from the SHEF-CSVD Study in which the baseline and follow-up CVR and MRI imaging could be evaluated (Staszewski et al., 2013). The study protocol with detailed selection criteria and methodology has been described previously (Staszewski et al., 2019).

In brief: the SVD group consisted of ambulatory subjects above 60 years, enrolled between December 2011 and September 2015, with established LS (according to the OCSP criteria), VaP, or $\mathrm{VaD}$ (according to the Hurtig or NINDS-AIREN criteria) (Chui et al., 1992; Hurtig, 1993; Zijlmans et al., 2004). The patients with MRI contraindications, non-SVD-related WMLs, strategic single-infarct dementia, or post-stroke $\mathrm{VaP}$ or $\mathrm{VaD}$, recurrent LS, carotid artery stenosis $\geq 50 \%$, atrial fibrillation, chronic kidney disease (CKD) requiring dialysis, life expectancy $<6$ months, and recent head trauma were not included. To maximize the statistical power, we decided to analyze the equal groups of subjects and we recruited the consecutive patients with $\mathrm{VaP}$ and matched them in a 1:1 ratio with $\mathrm{VaD}$, LS patients, and CG (without known cerebrovascular disease or dementia) according to sex, age ( \pm 5 years), and the presence of diabetes and hypertension. The signs of SVD are often seen in MRI in cognitively healthy elderly, and they are highly age-related. The studies showed that the WMLs are detected in 30-90\% of cognitively healthy elderly (mean 72-74 years), therefore to maximize the homogeneity of the studied groups, we included only controls with no radiological signs of SVD in MRI (Fazekas 0 ) and the patients with SVD with extensive WMLs (Fazekas grade 2 or 3) (Longstreth et al., 2005; Gustavsson et al., 2015).

Of the 139 screened subjects (101 patients with SVD and 38 controls), 59 were excluded (18 controls, $15 \mathrm{LS}, 9 \mathrm{VaD}$, and 17 $\mathrm{VaP})$ due to inadequate acoustical bone window at the baseline $(n=16)$, the radiological markers of SVD in controls at baseline MRI examination $(n=3)$, withdrawal of consent during followup $(n=7)$, lack of follow-up MRI $(n=22)$, or TCD examination $(n=11)$. The patients with SVD and incomplete follow-up data $(n=30)$ had similar mean age $(70.2 \pm 7.6$ vs. $72.6 \pm 6.9, p=$ $0.14)$ and the baseline SVD score $(2.1 \pm 0.6$ vs. $2 \pm 0.6, p=$ $0.6)$ comparing with those included to the final analysis, however, more men dropped-out in comparison with women (48 vs. $9 \%, p$ $<0.01)$. The most common reported reason for lack of follow-up 
was that the subjects felt asymptomatic or did not tolerate well to the baseline MRI or TCD examination. Finally, the study group comprised 60 patients with newly diagnosed symptomatic SVD (20 per group: VaP, VaD, and LS) and 20 controls. All the patients were functionally independent (modified Rankin Scale, mRS $\leq$ 3 and total Barthel Index $\geq 80$ ) and without severe dementia (Mini-Mental State Examination, MMSE $\geq 12$ ) (Schulc et al., 2015).

\section{Study Procedures}

All the patients signed informed consent before entering the study. The consent has been obtained prior to any study specific procedures. All the patients had TCD and MRI examinations performed at baseline and the 24-month follow-up visit (mean $23.1 \pm 4$ months; LS $22.5 \pm 3$; VaP $21.5 \pm 4.6$; VaD $23.3 \pm 3.4$; CG $23.6 \pm 2$; and ANOVA $p=0.28$ ).

\section{Ultrasound Examinations}

The determination of the cerebrovascular reserve capacity is based on the ability of the intracranial arterioles to dilate. The $\mathrm{CO}_{2}$ tests assume the correlation among the $\mathrm{CBF}$, the $\mathrm{CO}_{2}$ partial pressure, and the flow velocity in the basal cerebral arteries, and the reduction or elevation of $\mathrm{pCO}_{2}$ leads to a decrease or increase in MFV. Since TCD measures flow velocity in the large arteries, this reflects flow in the combined gray and white matter. The TCD study included evaluation of both the MCAs with 2-MHz probes (Companion III, Nicolet) in fixed positions according to the standard protocol (Settakis et al., 2002). The MFV values were averaged, and the interhemispheric differences for mean MFV did not exceed 15\%. The recordings were considered acceptable when the velocities of blood flow could be detected bilaterally, and with a clear envelope of the MFV spectrum during the entire cardiac cycle. The CVR was measured as the breath-holding index $(\mathrm{BHI})$, the ratio of the percentage MFV increase during hypercapnia, and vasomotor reactivity reserve (VMRr), the percentage change in $\mathrm{MFV}$ from hypo- to hypercapnia (Tsivgoulis and Alexandrov, 2008). Basing on the Markus and Harrison procedure, we measured the baseline MFV (baseMFV) following $10 \mathrm{~min}$ of rest with normal breathing (normocapnia), the minimal MFV value (minMFV) following $2 \mathrm{~min}$ of hyperventilation (hypocapnia), and the MFV value (maxMFV) subsequent to $30 \mathrm{~s}$ of breath holding (hypercapnia) and followed by a period of $4 \mathrm{~min}$ of normal breathing of room air (Markus and Harrison, 1992). To achieve the most reproducible results before proceeding to the definitive recording, the participants were trained to perform all the procedures correctly. All of them were able to hyperventilate and hold their breath for the required period. The exact length of breath-holding ranged from 29.8 to $30.5 \mathrm{~s}$ at the baseline and 30.1 to $30.3 \mathrm{~s}$ at follow-up, and it did not differ between the study groups (ANOVA, $p>0.1$ ). The end-tidal $\mathrm{CO}_{2}$ $(\text { etCO })_{2}$ concentration was monitored by capnograph (PC900A, Creative Medical, Shenzhen, China) during the examination. Blood pressure $(\mathrm{BP})$ and heart rate $(\mathrm{HR})$ were measured prior to and following the tests. Although McDonnell et al. found higher intra-rater reliability for the TCD measurements taken while the patients were sitting, we performed TCD examination in all the subjects in the supine position in accordance with our standard protocol (McDonnell et al., 2013). Ultrasound examination was performed under the standardized conditions (same quiet room and time of the day; no sleep deprivation and no medication intake for at least $6 \mathrm{~h}$ were allowed) by a single experienced TCD sonographer unaware of the diagnosis of the subjects. All the patients with LS had the study procedures performed at least 3 weeks (mean $24 \pm 2$ days) after their index strokes.

\section{MRI Evaluation (GE Healthcare 1.5 T Scanner, IL, USA)}

The images were evaluated for the presence of acute LS, lacunes, deep WMLs (dWMLs), or periventricular (pWMLs), microbleeds (MBs), and enlarged perivascular spaces (PVS) according to the STRIVE guidelines and visual SVD scale (Wardlaw et al., 2013; Staals et al., 2014). The simple modified Fazekas rating scale was used to estimate the extent of pWMLs and dWMLs (Fazekas et al., 1987; Inzitari et al., 2009). The mild white matter lesions (Fazekas grade 1) were defined as the punctate lesions in the deep white matter with a maximum diameter of $9 \mathrm{~mm}$ for every single lesion and $20 \mathrm{~mm}$ for grouped lesions. The moderate white matter lesions (grade 2) were defined as early confluent lesions of 10-20 mm for single lesions and $>20 \mathrm{~mm}$ for grouped lesions in any diameter, with no more than connecting bridges between the individual lesions. Severe white matter lesions (grade 3) were defined as single lesions or confluent areas of hyperintensity of $20 \mathrm{~mm}$ or greater in any diameter. The presence of each of the four MRI markers for SVD (WMH, lacunes, cerebral microbleeds, and perivascular spaces) was counted to retrieve a total SVD score (ranging from 0 to 4). One point on the visual SVD scale was awarded if confluent deep WMLs (Fazekas grade 2 and 3) or irregular periventricular hyperintensities extending into the deep white matter (Fazekas grade 3) were present or when one or more lacunes or MBs were present; 1 point was awarded if moderate (10-25) to extensive $(>25)$ enlarged PVS were present (Kim et al., 2008). The presence of each marker produced a score of a minimum 0 and a maximum of 4, representing the total MRI load of SVD. At baseline MRI assessment, all the patients with SVD had at least Fazekas grade 2 WMLs, and controls did not have radiological markers of SVD in MRI. The visual rating of SVD radiological progression expressed by the WMLs progression or development of new lacunes was performed at a follow-up visit. As proposed by Prins et al., the WMLs progression and lacunes were rated on the FLAIR images and the presence or absence of progression was rated in the three periventricular regions, basal ganglia, infratentorial region, and four subcortical white matter regions (Prins et al., 2004). The images were reviewed by a neuroradiologist (E.S.) blinded to the clinical data.

\section{Atherothrombotic Risk Evaluation}

The vascular comorbidities were defined according to the current standards and evaluated based on the available medical data, physical examinations, widespread histories, and routine laboratory tests performed at baseline (Alberti et al., 2009; Goblirsch et al., 2013). 


\section{Statistical Analysis}

All the demographic data were summarized, tabulated, and verified for normality with the Shapiro-Wilk test, and the homogeneity of the variances was assured by Levene's test. Categorical and continuous data are presented as frequencies or means $\pm \mathrm{SD}$ and analyzed using Fisher's exact tests, the chi-square test, paired $t$-tests, or non-parametric tests where appropriate.

The associations between MRI progression with CVR changes over time in the studied groups were compared using a linear mixed model. The model included study group (SVD, CG), Time (Baseline, Follow-up), MRI (progression, no progression), and a Time*Group*MRI interaction for fixed effects. The subject variable was specified to assess possible individual variability. Per group, the Wilcoxon's signed-rank test was performed to evaluate the differences between CVR and other hemodynamic measures at the baseline and at follow-up visits. One-way ANOVA and chisquare tests were used to compare data between the study groups with post-hoc Tukey's honestly significant difference (HSD) tests for comparisons among the SVD subgroups. The effect sizes for the group differences were analyzed with partial eta squared $\left(\eta_{\mathrm{p}}^{2}\right)$ reflecting the proportion of the total variance attributable to the effect (and considered small if 0.01 , moderate around 0.06 , and high if $>0.13$ ). A probability value of $p<0.05$ was

TABLE 1 | Main characteristics of the study population at baseline.

\begin{tabular}{|c|c|c|c|c|c|c|}
\hline Variable & SVD $(n=60)$ & LS $(n=20)$ & $\operatorname{VaP}(n=20)$ & $\operatorname{VaD}(n=20)$ & $C G(n=20)$ & $p^{\#}$ \\
\hline \multicolumn{7}{|l|}{ Demographics } \\
\hline Age $(y)^{\star}$ & $72.6( \pm 6.9)$ & $71.7( \pm 7.7)$ & $73.8( \pm 6.2)$ & $72.2( \pm 6.8)$ & $71.9( \pm 3.2)$ & 0.7 \\
\hline \multicolumn{7}{|l|}{ Vascular risk factors } \\
\hline Hypertension (\%) ${ }^{*}$ & $54(89)$ & $18(90)$ & $18(90)$ & $18(90)$ & $18(90)$ & 1 \\
\hline Current smoking (\%) & $22(37)$ & 7 (35) & $6(30)$ & $9(45)$ & $8(40)$ & 0.5 \\
\hline Hyperlipidemia (\%) & $45(75)$ & $15(75)$ & $16(80)$ & $14(70)$ & $15(75)$ & 0.8 \\
\hline CKD (\%) & $8(13)$ & $2(10)$ & $2(10)$ & $4(20)$ & $3(15)$ & 0.3 \\
\hline PS (\%) & $28(46)$ & $10(50)$ & $10(50)$ & $8(40)$ & $8(40)$ & 0.8 \\
\hline Obesity (BMl>30) & $17(28)$ & $6(30)$ & $6(30)$ & $5(25)$ & $6(30)$ & 0.8 \\
\hline Uric acid (mg/dL) & $5.4 \pm 1.5$ & $5.8 \pm 1.6$ & $5.5 \pm 1.3$ & $4.8 \pm 1.5$ & $4.6 \pm 1.3$ & 0.05 \\
\hline eGFR (ml/min/1.73 m²) & $78.8 \pm 24.1$ & $77.1 \pm 29$ & $72.3 \pm 22.8$ & $87 \pm 17.2$ & $95 \pm 16$ & 0.01 \\
\hline HbA1c (\%) & $6.2 \pm 1.03$ & $6.3 \pm 1.2$ & $5.9 \pm 0.5$ & $6.3 \pm 1.2$ & $5.9 \pm 0.1$ & 0.26 \\
\hline $\mathrm{FG}(\mathrm{mg} / \mathrm{dL})$ & $120.1 \pm 43.2$ & $128.8 \pm 48.6$ & $112.8 \pm 32.3$ & $120.6 \pm 47.2$ & $106.3 \pm 18.7$ & 0.21 \\
\hline LDL (mg/dL) & $108.4 \pm 37.8$ & $97.1 \pm 35.5$ & $110.6 \pm 36.6$ & $116.5 \pm 40.4$ & $122.8 \pm 41.8$ & 0.22 \\
\hline $\mathrm{HDL}(\mathrm{mg} / \mathrm{dL})$ & $52.2 \pm 17$ & $46.7 \pm 10$ & $55.2 \pm 16.9$ & $54.1 \pm 22.5$ & $60.9 \pm 19.3$ & 0.12 \\
\hline $\mathrm{TG}(\mathrm{mg} / \mathrm{dL})$ & $124.5 \pm 57.9$ & $125.6 \pm 60.1$ & $132.1 \pm 64.2$ & $115.5 \pm 50$ & $115.1 \pm 39$ & 0.7 \\
\hline TC (mg/dL) & $185.2 \pm 48.1$ & $166.8 \pm 39.5$ & $194.4 \pm 54.7$ & $193.4 \pm 54.7$ & $198.7 \pm 31.7$ & 0.1 \\
\hline BMl & $26.4 \pm 5.6$ & $27.7 \pm 7.6$ & $25.1 \pm 4$ & $26.3 \pm 4.5$ & $27.8 \pm 3.8$ & 0.3 \\
\hline \multicolumn{7}{|l|}{ MRI examination } \\
\hline SVD score (mean \pm SD) & $2 \pm 0.6$ & $2.2 \pm 0.7$ & $2 \pm 0.6$ & $2.1 \pm 0.6$ & 0 & $<0.01$ \\
\hline Calcium channel blocker (\%) & $23(38)$ & $9(40)$ & 7 (35) & 7 (35) & $7(35)$ & 0.4 \\
\hline Diuretics (\%) & $12(20)$ & $4(20)$ & $3(15)$ & $5(25)$ & $4(20)$ & 0.2 \\
\hline
\end{tabular}

Values represent the means ( $\pm S D$ ) for continuously distributed data or the numbers (\%) for categorical data.

"Matched factors; " ANOVA and $\chi^{2}$ difference between the groups $p<0.05$ compared with CG according to Tukey's honestly significant difference (HSD) test.

SVD, cerebral small vessel disease; LS, lacunar stroke; VaD, vascular dementia; VaP, vascular parkinsonism; CG, control group; CAD, coronary artery disease; BMI, body mass index; FG, fasting glucose; PS, polymetabolic syndrome; CKD, chronic kidney disease; IMT, intima-media thickness; ACEl, angiotensin-converting-enzyme inhibitors, ARB, angiotensin receptor blockers. 
considered significant, all the analyses were made using the PQStat 1.8.0 software.

The study has been approved by the Internal Revision Board (Wojskowy Instytut Medyczny, 46/WIM/2010) and it was conducted in accordance with the Declaration of Helsinki.

\section{RESULTS}

The studied cohort consisted of 80 older adults (mean $72.4 \pm 6.2$ years) of both sexes ( $50 \%$ women). The baseline characteristic of the studied cohort is provided in Table 1. The prevalence of the main comorbidities was similar in groups, but there were some differences in the laboratory examination findings: the CG had lower concentrations of homocysteine, showed a trend toward lower concentration of uric acid, and had higher levels of estimated glomerular filtration rate (eGFRs) comparing with other subjects. The SVD subgroups had a similar prevalence of vascular risk factors and treatment use, laboratory, and MRI findings (Fazekas grade 3 was scored by $80 \% \mathrm{LS}, 80 \% \mathrm{VaD}$, and $75 \%$ VaP subjects; $p=0.7)$. The mean etCO $\mathrm{C}_{2}$ concentrations and systolic blood pressure (SBP), diastolic blood pressure (DBP), and heart rate (HR) measures during hyperventilation and breath holding did not differ between the CG and the SVD group, and they were also similar between the SVD subgroups. In addition, these variables did not significantly differ between the baseline and follow-up assessments (Wilcoxon's sign rank test, $p>0.1$ ). The reproducibility measurements of $\mathrm{VMRr}$ and $\mathrm{BHI}$ were performed in a sample of 12 individuals (four from CG, eight with LS). The intraclass correlation coefficient for the two consecutive measurements was $0.90 ; p<0.01$ for $\mathrm{VMRr}$ and 0.88 ; $p<0.01$ for BHI.

Vasomotor reactivity reserve and the BHI were more severely impaired at the baseline and at follow-up assessments in the patients in the SVD groups (regardless of the clinical spectrum of the disease) than in the CG (Table 2). The size effect of the difference was similar in all comparisons (etasquared ranged between 0.25 and 0.29 ). For better visualization, the CVR change in the individual subjects is shown in (Supplementary Figures 1, 2).

Vasomotor reactivity reserve and the BHI were significantly lower in diabetic patients with SVD vs. diabetic controls at the two timepoints (baseline VMRr: $55.8 \pm 16$ vs. $74 \pm 18 \%$, respectively, $p<0.001$; $\mathrm{BHI}: 0.79 \pm 0.39$ vs. $1.1 \pm 0.3, p=$ 0.02 ; follow-up VMRr $52.7 \pm 15$ vs. $71.2 \pm 20, p<0.01$; BHI: $0.72 \pm 0.4$ vs. $1.04 \pm 0.5, p<0.01$ and the difference was also significant between the non-diabetic subjects from SVD group vs. CG (respectively; baseline VMRr: $54.2 \pm 18.4$ vs. $79.9 \pm 13.7, p$ $<0.001$; BHI: $0.78 \pm 0.39$ vs. $1.16 \pm 0.55, p=0.01$; follow-up VMRr $52.1 \pm 18$ vs. $76.8 \pm 14.9 \%, p<0.01$; BHI: $0.70 \pm 0.35$ vs. $1.15 \pm 0.2 ; p<0.001)$.

Both the assessed CVR measures (VMRr and the BHI) significantly declined over time in the subjects with SVD (Wilcoxon's signed-rank test, $p=0.01$ ), however, there was no significant decline of $\mathrm{VMRr}$ and $\mathrm{BHI}$ in $\mathrm{CG}$ over 24 months of observation (Wilcoxon's signed-rank test $p$ $>0.1$ ) (Figure 1). The mean VMRr and BHI values were also similar at the baseline and follow-up measurements

TABLE 2 | Basal and follow-up characteristics and vasodilatation responses of cerebral arteries in all the subjects.

\begin{tabular}{|c|c|c|c|c|c|c|}
\hline Variable & $\operatorname{SVD}(n=60)$ & $\operatorname{LS}(n=20)$ & $\operatorname{VaP}(n=20)$ & $\operatorname{VaD}(n=20)$ & CG $(n=20)$ & $p^{\#}$ \\
\hline \multicolumn{7}{|l|}{ Baseline measures } \\
\hline MFV (cm/s) & $41.5 \pm 12^{\star}$ & $43.9 \pm 15$ & $42 \pm 9.5$ & $38.4 \pm 10.4^{*}$ & $46.8 \pm 8.7$ & 0.03 \\
\hline $\operatorname{VMRr}(\%)$ & $56.7 \pm 18.4^{\star}$ & $55.4 \pm 18.6^{\star}$ & $55.1 \pm 15.2^{\star}$ & $54.2 \pm 18.7^{\star}$ & $77.2 \pm 15.6$ & $<0.001$ \\
\hline $\mathrm{BHI}$ & $0.82 \pm 0.39^{\star}$ & $0.86 \pm 0.4^{\star}$ & $0.77 \pm 0.28^{\star}$ & $0.71 \pm 0.4^{\star}$ & $1.15 \pm 0.47$ & $<0.001$ \\
\hline Resting SBP, mmHg & $127.2 \pm 17.2$ & $130.1 \pm 16.2$ & $126.1 \pm 11.1$ & $125.2 \pm 16$ & $124 \pm 19$ & 0.2 \\
\hline Resting DBP, mmHg & $73.4 \pm 10.1$ & $72.4 \pm 13.2$ & $73.2 \pm 12.4$ & $74.4 \pm 11$ & $74.1 \pm 8$ & 0.7 \\
\hline Resting HR, beats/min & $76.8 \pm 6.6$ & $76.8 \pm 4.7$ & $75.2 \pm 8.2$ & $78.3 \pm 6.6$ & $72.1 \pm 6.1$ & 0.8 \\
\hline Delta etCO 2 HV (\%) & $1.3 \pm 0.15$ & $1.4 \pm 0.4$ & $1.3 \pm 0.2$ & $1.1 \pm 0.7$ & $1.4 \pm 0.5$ & 0.6 \\
\hline Delta etCO 2 BH (\%) & $1.2 \pm 0.1$ & $1.3 \pm 0.2$ & $1.3 \pm 0.6$ & $1.1 \pm 0.6$ & $1.2 \pm 0.4$ & 0.8 \\
\hline \multicolumn{7}{|l|}{ Follow-up measures } \\
\hline MFV (cm/sek) & $35.2 \pm 11.2^{\star}$ & $37.1 \pm 15.6$ & $34.3 \pm 9.2^{\star}$ & $34.2 \pm 8.3^{\star}$ & $43.1 \pm 8.2$ & 0.06 \\
\hline VMRr (\%) & $52.3 \pm 16.7^{\star}$ & $54.4 \pm 17.9^{\star}$ & $49.3 \pm 11.5^{\star}$ & $53.2 \pm 19.7^{\star}$ & $74.3 \pm 17.6^{\star}$ & $<0.001$ \\
\hline $\mathrm{BHI}$ & $0.71 \pm 0.38^{\star}$ & $0.72 \pm 0.5^{\star}$ & $0.71 \pm 0.24^{\star}$ & $0.69 \pm 0.4^{\star}$ & $1.11 \pm 0.4^{*}$ & $<0.001$ \\
\hline Resting SBP, mmHg & $124.8 \pm 13.9$ & $127.2 \pm 17.1$ & $125.3 \pm 9.8$ & $122.1 \pm 15$ & $127 \pm 9$ & 0.3 \\
\hline Resting DBP, mmHg & $76.3 \pm 11$ & $74.1 \pm 14.1$ & $78.1 \pm 7.7$ & $76.7 \pm 11$ & $74.2 \pm 10$ & 0.6 \\
\hline Resting HR, beats/min & $73.9 \pm 2.2$ & $72.6 \pm 3$ & $75.2 \pm 1.2$ & $74.1 \pm 2.4$ & $70 \pm 10.5$ & 0.2 \\
\hline Delta etCO 2 HV (\%) & $1.23 \pm 0.6$ & $1.3 \pm 0.7$ & $1.2 \pm 0.5$ & $1.2 \pm 0.6$ & $1.9 \pm 0.7$ & 0.3 \\
\hline Delta etCO 2 BH (\%) & $1.16 \pm 0.7$ & $1.1 \pm 0.8$ & $1.1 \pm 0.4$ & $1.3 \pm 0.4$ & $1.2 \pm 0.7$ & 0.8 \\
\hline
\end{tabular}

\#ANOVA difference between the groups, ${ }^{*} p<0.05$ compared with the CG.

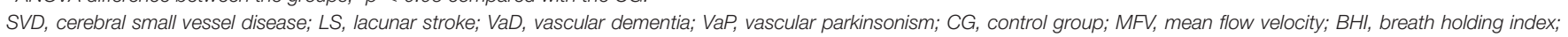
$V M R$, vasomotor reactivity reserve; PI, pulsatile index; RI, resistance index; SBP, systolic blood pressure; DBP, diastolic blood pressure; HR, heart rate.

$\Delta$ etCO $\mathrm{CO}_{2} \mathrm{H}$ end-tidal $\mathrm{CO}_{2}$ during hyperventilation; $\Delta$ etCO $\mathrm{BH}, \Delta$ end-tidal $\mathrm{CO}_{2}$ during breath holding. 


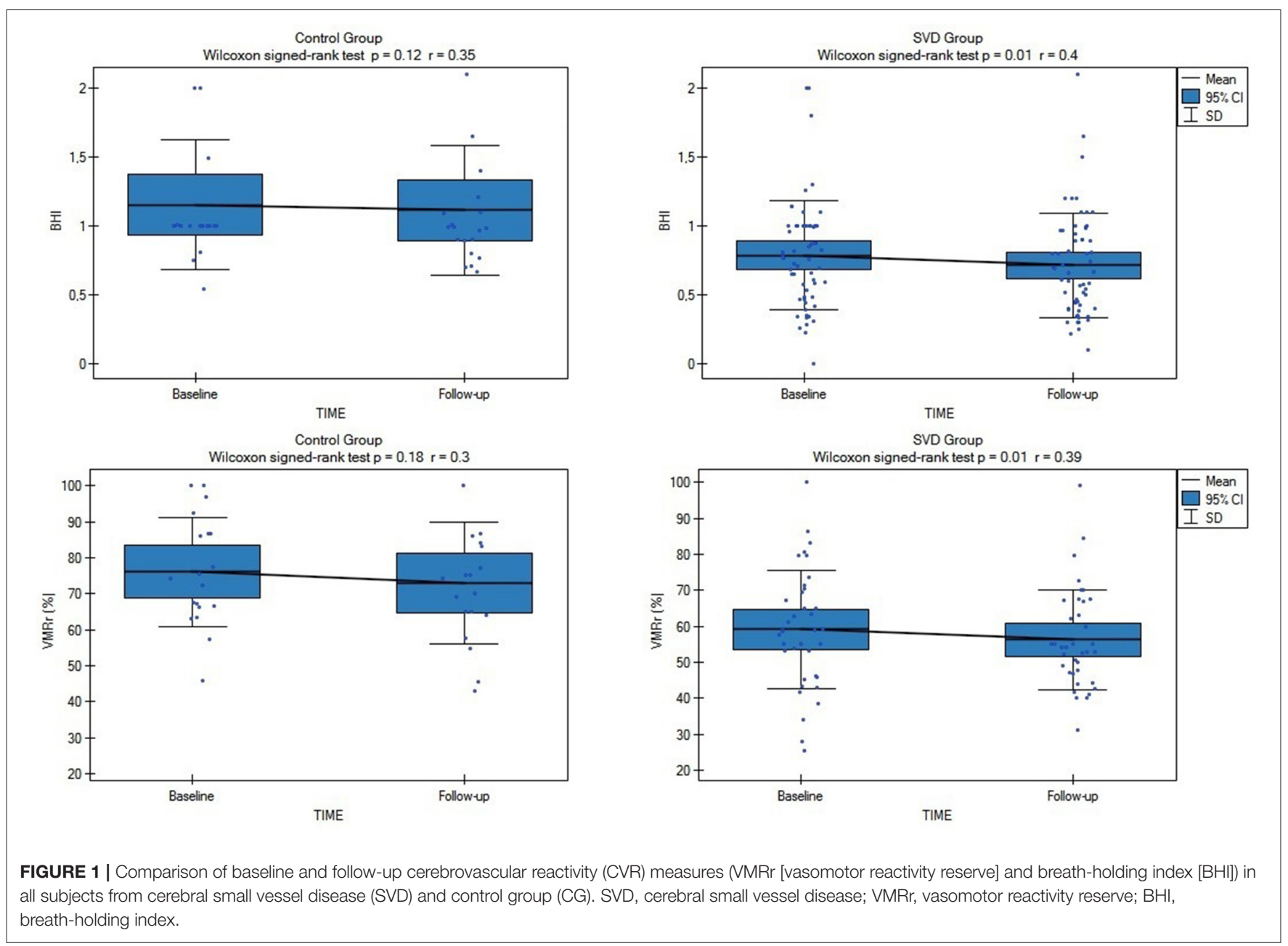

among the patients with LS, VaD, and VaP (ANOVA, $p>0.1$.

Radiological progression during the study was observed in 28 of the 80 subjects (35\%): significantly more often $(p=0.03)$ in the SVD subjects $(n=25$ [42\%]: eight subjects from LS [40\%] group, eight with $\mathrm{VaD}$ [40\%], and nine with $\mathrm{VaP}$ [45\%], with no difference between the SVD groups [ANOVA $p>0.1]$ ) than in the CG $(n=3 ; 15 \%)$. During the follow-up, only $4 / 80$ patients (5\%) experienced lacunar stroke (two patients from LS group and two from VaD group), no other vascular events were observed. Although in patients with SVD who experienced radiological progression, all the CVR measures were considerably impaired at the baseline and follow-up assessments compared with the no progression group (respectively, baseline VMRr $43.3 \pm 13.2 \%$ vs. $63.3 \pm 14.9 \%, p<0.001$; eta-square 0.33 ; baseline BHI $0.63 \pm$ 0.39 vs. $0.9 \pm 0.36, p<0.001$; eta-square 0.12 ; follow-up $\mathrm{VMRr}$ $41.2 \pm 13.4 \%$ vs. $60.3 \pm 14 \%, p<0.001$; eta-square 0.32 ; BHI 0.52 \pm 0.24 vs. $0.84 \pm 0.39, p<0.001$; eta-square 0.19 ), no significant decrease of CVR measures was observed during 24 months of observation (Wilcoxon's signed-rank test, $p>0.1$ ) (Figure 2). These data did not change significantly when we analyzed all the patients (SVD and CG) with the radiological progression vs. no progression group (respectively, baseline VMRr $45.4 \pm 14.1 \%$ vs. $68.6 \pm 16.9 \%$, $p<0.001$; baseline BHI $0.65 \pm 0.37$ vs. 1.0 $\pm 0.42, p<0.001$; follow-up VMRr $43.1 \pm 14.3 \%$ vs. $65.7 \pm$ $16.9 \%, p<0.001$; BHI $0.55 \pm 0.24$ vs. $1.0 \pm 0.47, p<0.001)$ and there was also no significant decrease of CVR measures over time of observation (Wilcoxon's signed-rank test, $p>0.1$ ). No interaction between CVR measures, time of assessment, and radiological progression in the SVD group were observed in the fixed linear analysis (Table 3).

\section{DISCUSSION}

This prospective study performed in symptomatic SVD has shown that the change in CVR measures is detectable following a 24-month period and that $42 \%$ of patients with SVD demonstrated radiological progression. We also confirmed that the cerebral vasoreactivity measures decreased in SVD regardless of the clinical manifestation of the disease. On the opposite, we did not demonstrate a significant CVR decrease in the subjects from CG who were neurologically asymptomatic, had no radiological markers of SVD in the baseline MRI, but who shared similar atherothrombotic risk factors as the SVD group. Although patients with SVD and radiological progression had 


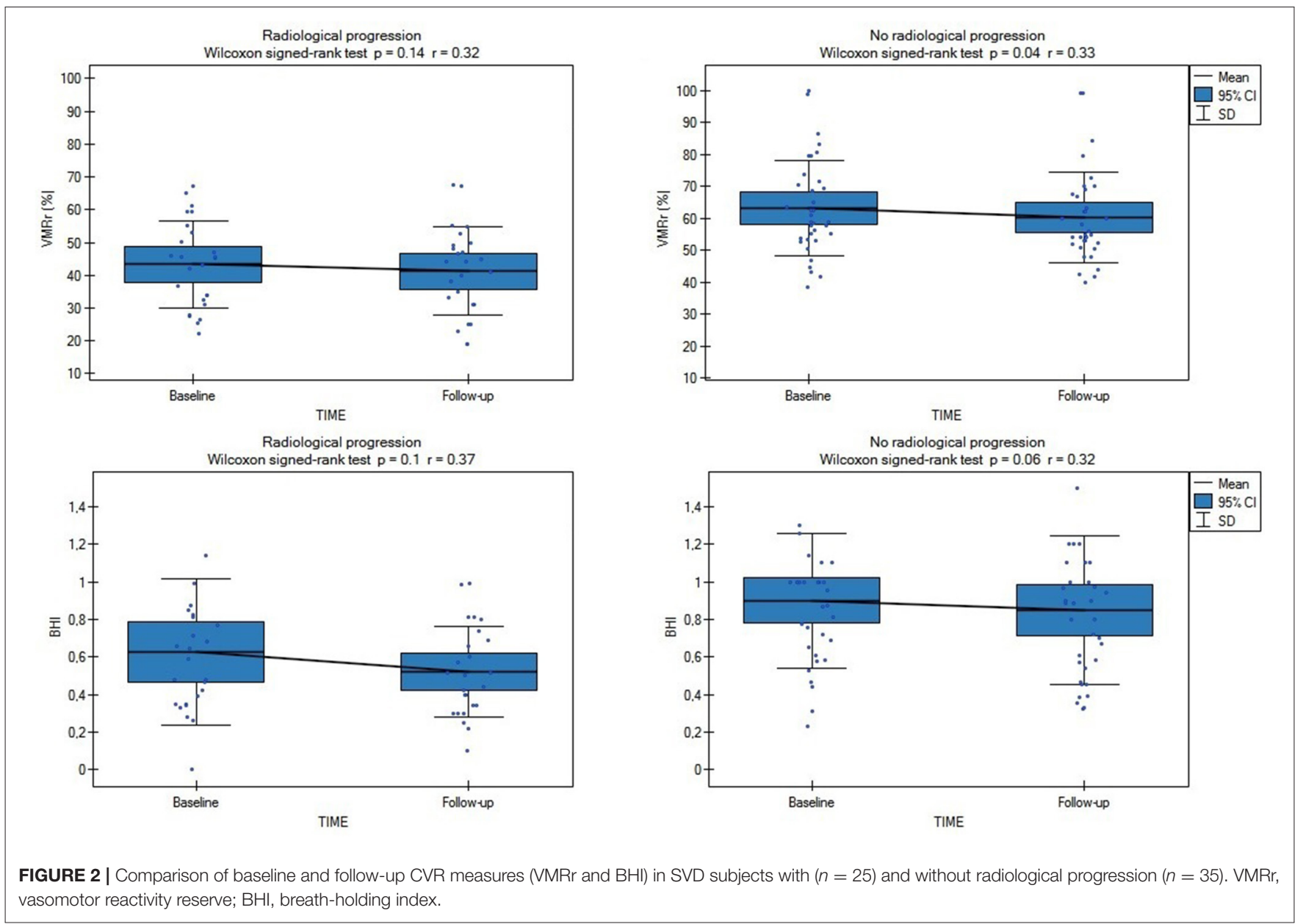

TABLE 3 | Association among the cerebral small vessel disease (SVD), assessment time point, and MRI progression on the cerebrovascular reactivity (CVR) measures.

\begin{tabular}{|c|c|c|c|c|c|c|c|}
\hline \multirow[b]{2}{*}{ Dependent variable } & \multirow[b]{2}{*}{ Fixed factor(s) } & \multirow[b]{2}{*}{ B } & \multirow[b]{2}{*}{ SE } & \multirow[b]{2}{*}{$t$} & \multirow[b]{2}{*}{$P^{*}$} & \multicolumn{2}{|c|}{$95 \% \mathrm{Cl}$} \\
\hline & & & & & & Lower limit & Upper limit \\
\hline \multicolumn{8}{|l|}{ BHI } \\
\hline & Time & -0.01 & 0.05 & -0.01 & 0.90 & -0.11 & 0.11 \\
\hline & SVD & -0.47 & 0.11 & -4.26 & $<0.01$ & -0.69 & -0.25 \\
\hline & MRI progression & -0.60 & 0.22 & -2.70 & 0.01 & -1.04 & -0.16 \\
\hline & Time ${ }^{\star}$ SVD & 0.05 & 0.07 & 0.55 & 0.50 & -0.20 & 0.38 \\
\hline & Time*MRI progression & 0.08 & 0.18 & 1.20 & 0.24 & -0.15 & 0.59 \\
\hline & SVD*MRI progression & 0.29 & 0.24 & 1.23 & 0.22 & -0.18 & 0.77 \\
\hline & Time*SVD*MRI progression & -0.02 & 0.16 & -0.13 & 0.80 & -0.34 & 0.30 \\
\hline \multicolumn{8}{|l|}{ VMRr } \\
\hline & Time & -3.90 & 2.60 & -1.40 & 0.20 & -9.20 & 1.30 \\
\hline & SVD & -27.10 & 4.34 & -6.20 & $<0.01$ & -35.70 & -18.50 \\
\hline & MR progression & -28.80 & 9.20 & -3.10 & 0.01 & -47.10 & -10.50 \\
\hline & Time ${ }^{\star} S V D$ & 7.05 & 3.23 & 2.13 & 0.20 & -0.20 & 5.97 \\
\hline & Time*MRI progression & 9.04 & 6.80 & 1.32 & 0.20 & -4.50 & 22.60 \\
\hline & SVD*MRI progression & 9.80 & 9.90 & 0.90 & 0.30 & -9.98 & 29.60 \\
\hline & Time*SVD*MRI progression & -10.07 & 7.40 & -1.35 & 0.17 & -24.80 & 6.70 \\
\hline
\end{tabular}

* Linear mixed effects model.

BHI, breath holding index; VMRr, vasoreactivity reserve; SVD, small vessel disease. 
TABLE 4 | Major transcranial Doppler ultrasound (TCD) studies assessing CVR in sporadic small vessel disease populations.

\begin{tabular}{|c|c|c|c|}
\hline References & Study population & CVR assessment & Results \\
\hline Molina et al. (1999) & $\begin{array}{l}46 \text { patients with lacunar stroke } \\
\text { and } 46 \mathrm{CG}\end{array}$ & Acetazolamide test & Significant CVR reduction in SVD group \\
\hline Terborg et al. (2000) & 46 patients with SVD and 13 CG & $\begin{array}{l}\text { Ventilation tests; } \\
\text { NIRS }\end{array}$ & $\begin{array}{l}\text { Significantly reduced VMR in TCD and NIRS assessments in severe } \\
\text { SVD; a good correlation between the validity of TCD and NIRS }\end{array}$ \\
\hline Kidwell et al. (2001) & 55 patients with SVD & $\mathrm{Pl}$ & $\begin{array}{l}\text { Correlation between PI and severity of SVD ; PI cut points allowed } \\
\text { discrimination of PVH with } 89 \% \text { sensitivity and } 86 \% \text { specificity and } \\
\text { discrimination of DWMH with } 70 \% \text { sensitivity and } 73 \% \text { specificity. }\end{array}$ \\
\hline Pánczél et al. (2002) & $\begin{array}{l}25 \text { patients with lacunar stroke, } \\
20 \text { patients with leukoaraiosis } \\
\text { and } 35 \text { CG }\end{array}$ & $\begin{array}{l}\text { Tilting, ventilation, } \\
\text { and acetazolamide } \\
\text { tests }\end{array}$ & $\begin{array}{l}\text { CVR in BA and MCA territory was impaired in hypercapnia in SVD; no } \\
\text { significant differences between CVR measures in BA and MCA territory } \\
\text { in acetazolamide test; significantly higher } \mathrm{VRCO}_{2} \text { in } \mathrm{MCA} \text { vs BA } \\
\text { measurements }\end{array}$ \\
\hline Ghorbani et al. (2015) & 56 patients with SVD and 48 CG & $\mathrm{Pl}$ & $\begin{array}{l}\text { Good correlation between } \mathrm{PI} \text { and SVD radiological manifestations: } \\
\text { - In PVH with PI cut-off }=0.83 \text {, the sensitivity } 90 \% \text { and specificity } 98 \% \\
\text { - In DWMH with } \mathrm{PI}=0.79 \text {, the sensitivity } 75 \% \text { specificity } 87.5 \% \\
\text { - In lacunar stroke with } \mathrm{PI}=0.80 \text {, the sensitivity } 73 \% \text { and } \\
\text { specificity } 90 \% \text {. } \\
\text { - In } \mathrm{PH} \text { with } \mathrm{PI}=0.69 \text {, the sensitivity } 92 \% \text { and specificity } 87.5 \% \text {. } \\
\text { - In PVH+ DWMH+ lacunar with } \mathrm{PI}=0.83 \text {, the sensitivity } 90 \% \text { and } \\
\text { specificity } 96 \% \text {. }\end{array}$ \\
\hline Nam et al. (2020) & 206 patients with lacunar stroke & $\mathrm{Pl}$ & $\begin{array}{l}\text { PI was positively associated with the WMHs volume and the presence } \\
\text { of old lacunar infarcts. }\end{array}$ \\
\hline
\end{tabular}

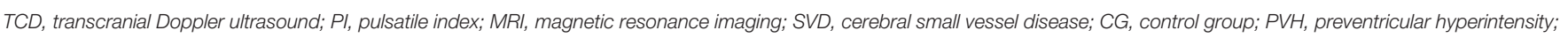

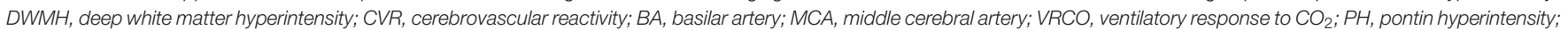
NIRS, near-infrared spectroscopy; CT, computed tomography.

severely impaired CVR at the baseline and follow-up comparing with the subjects with no MRI progression, there was no significant interaction between that CVR decline and risk of radiological progression.

Besides of growing evidence of MRI techniques which increasingly play a more important role as a non-invasive tool capable to assess the cerebral reserve capacity in combination with a vascular challenge, the vasomotor reactivity testing with TCD is still a gold diagnostic standard and it has been proved to assess $\mathrm{CBF}$ and indirectly monitor the function of ED in patients with cerebral microangiopathy (Kozera et al., 2009; D'Andrea et al., 2016). The TCD studies demonstrated that CVR is negatively correlated with the duration of hypertension, patient age, and history of cerebrovascular events (Fujishima et al., 2001; Kozera et al., 2010). Some older studies using SPECT or Xenon CT techniques revealed that LS, particularly with leukoaraiosis was associated with the reduced CBF in the white matter and cortex in the subjects with severe WMLs, while the studies on $\mathrm{VaD}$ consistently showed reductions in both the white and gray matter (Markus et al., 2000). Many studies investigated CBF in the patients with SVD using TCD techniques, and in line with our data, the majority of them revealed impaired $\mathrm{CBF}$ comparing with the healthy controls in the patients with symptomatic and asymptomatic LS, more marked in multiple comparing with single infarcts and a reduction in reactivity that was correlated with the degree of leukoaraiosis (Table 4) (Maeda et al., 1993; Molina et al., 1999; Terborg et al., 2000). There is, however, a lack of studies on the dynamic CVR changes over time, especially in SVD, and in well-characterized SVD populations, e.g., in rarely studied patients with $\mathrm{VaD}$ and $\mathrm{VaP}$. As SVD is a wide term and it is used to describe the different disease processes ranging from asymptomatic WMLs in normal individuals through to the symptomatic SVD subjects, as we aimed in the presented report, our data added some further evidence that cerebral ED occurs and progresses in patients with LS, VaD, or VaP due to SVD, even if they had well-controlled hypertension (according to the baseline and follow-up BP measurements). Interestingly, a similar trend was not observed in CG with comparable atherothrombotic risk factors (age, sex, diabetes, hypertension, obesity, and smoking), and this may suggest that other vascular factors or duration of exposure to vascular risk factors in SVD are more important and responsible for a continuous decline in the CVR (Muoio et al., 2014). For example, the studies on patients with cerebrovascular disease showed that the reduction in vasodilatory capacity in asymptomatic carotid disease can predispose for the development of cerebrovascular disease (Silvestrini et al., 2000). In 2020, Soman et al. (2020) using arterial spin labeling MRI to evaluate which cardiovascular risk factors alter the CVR found that higher SBP, chronic kidney disease, history of past stroke, and hypercholesterolemia are responsible for the impaired CVR. Although the controls and patients with SVD in our study were well-balanced with the main comorbidities, we did not control other important risk factors of SVD. For instance, chronic poor sleep patterns, depression, prediabetes, and unhealthy diet (dietary salt) can impact the endothelial function through immune influences (Hakim, 2019). Of notice, the Oxford Vascular (OXVASC) Study importantly showed that premorbid hypertension in midlife correlated more strongly with the global SVD burden than a single blood pressure measurement or a known history of hypertension (Lau et al., 2018). 
Whether the progressive decrease of CVR is specific to the progressive nature of SVD is unknown (Stevenson et al., 2010). Importantly, we showed that there was no difference in the CVR at the baseline and follow-up assessments between the SVD clinical subgroups and this finding possibly could be related to similar WMLs burden or comparable prevalence of comorbidities. We also observed that the subjects with radiological progression had the most impaired CVR at baseline which, however, did not significantly decrease over time. These findings may indicate that MRI progression in that group may be related to the baseline severe CVR impairment or other factors, e.g., inflammatory or prothrombotic. It is also possible that the TCD measures are less sensitive to the more subtle changes, particularly in the white matter flow. Our results are, however, opposite to a large study in 628 asymptomatic individuals (mean 69 years) with WMLs which showed a progressive reduction in the CBF velocity measured using TCD as WMLs severity increased (Tzourio et al., 2001). Some similar data were demonstrated by Sam et al., who established areas of reduced CVR that preceded the development of WMLs by advanced MRI techniques, suggesting that CVR impairment contributes to the development and progression of SVD (Sam et al., 2016). On the opposite, in a PROSPER trial of statin therapy that investigated 390 individuals with cardiovascular risk factors, there was no association between $\mathrm{CBF}$ and the prevalence of WMLs at baseline, a decline of CBF, and risk of development of deep WMLs (Ten Dam et al., 2007). Our results are also in line with the study that showed significantly reduced CVR in LS than in the CG, which, however, could not be explained by the main atherothrombotic risk factors (Deplanque et al., 2013). It is important to appreciate that cerebral reactivity and autoregulation are not fully understood, although the neurogenic, metabolic, or myogenic factors were proposed. Of notice, $\mathrm{CO}_{2}-\mathrm{CVR}$ is only one of the several mechanisms of cerebral autoregulation, thus preserved $\mathrm{CO}_{2}$ CVR does not imply intact cerebral autoregulation (Klinzing et al., 2021). Therefore, CVR impairment could be due to different mechanisms, e.g., related to genetic factors or BBB dysfunction, resulting in microvascular dysfunction (Zlokovic, 2011). Some important aspects of the present study related to the patients with $\mathrm{VaD}$ and $\mathrm{VaP}$ should be underlined as these subjects probably share SVD pathology with neurodegenerative disease, and it is not established whether the microvascular changes reflect the diminished demand caused by the advanced neurodegeneration or whether SVD precedes and contributes to the neurodegeneration. For example, impaired cerebrovascular reserve capacity and vasoreactivity (identified using TCD along with the $\mathrm{BHI}$ ) was found in the patients with mild cognitive impairment, Alzheimer's disease, and Parkinson's disease without severe underlying atherosclerosis (Shim et al., 2015; Urbanova et al., 2018). Although the contribution of CVR impairment to the pathogenesis of neurodegenerative diseases is not certain, it might be suspected that a reduced cerebrovascular reserve is an additional deteriorating factor in the neurodegeneration (Ojeda et al., 2017). One unanswered key question is that whether any reductions in the $\mathrm{CBF}$ are primary, or merely occurs secondary to the brain damage as due to vaso-neuronal coupling, the reductions in brain metabolism are associated with reduced CBF (Markus et al., 2014).

Most studies in the literature have demonstrated the differences in CBF between the groups of patients with SVD and controls, with very few studies addressing the sensitivity and specificity of these parameters in TCD and MRI examinations (Panerai, 2009). The large variations in CVR across the patients and between different sessions of the same subject are often observed in the TCD and MRI studies, which hamper the ability of these measures in monitoring disease progression (Hou et al., 2020). It should be especially stressed that there are concerns with the application of the $\mathrm{BHI}$ as the relationship between breath-hold length and the $\mathrm{pCO}_{2}$ stimulus remains unclear and this test has low reproducibility and high variability (Alwatban et al., 2018; Koep et al., 2020). The latest studies showed that the blood oxygenation level dependent MRI (BOLDMRI) method is most reliable and reproducible because it has the advantage of mapping the whole brain with good spatial resolution, allowing investigation of CVR regional distribution (Leoni et al., 2012). Despite the relationships between the baseline CBF measures from TCD and MRI, the current studies showed no direct correlations between the CVR metrics calculated from TCD and MRI-BOLD measures during a $5 \% \mathrm{CO}_{2}$ challenge (Burley et al., 2021b). These variations in the CVR measures in different imaging modalities are important as they significantly reduce the statistical power to detect the pathologyrelated differences, preclude personalized determination of abnormalities, and challenge the validity of comparing CVR metrics across the studies.

Our study has several limitations, and the data should be cautiously interpreted owing to the single-center design and a small number of subjects included that may introduce bias. Even if we analyzed a relatively homogeneous cohort and highly selected SVD sample of patients, it should be considered a hypothesis-generating pilot study, because it may be less representative of the overall SVD population. However, the majority of the TCD studies on CVR in subjects with SVD had low patient numbers or controls (Table 4). Although the patients were prohibited from taking any drugs before the examinations, some medications could have affected the dilatory responses of the arteries. In future studies, additional research may be needed to determine the effect of treatment as a function of CBF on the prognosis of patients. The other limitations are that we used 1.5 Tesla MRI which potentially limited the accuracy of the radiological assessment, and we did not account for etCO $\mathrm{CO}_{2}$ for calculating the CVR. This could have influenced the results and limit the generalizability and reproducibility of the study, since $\mathrm{PaCO}_{2}$ has a marked influence on $\mathrm{CBF}$ and autoregulation, evaluations could be compromised in situations where significant changes in $\mathrm{PaCO}_{2}$ are undetected (Panerai et al., 1999). However, in our study, no significant difference between the subgroups and between the baseline and follow-up etCO $\mathrm{C}_{2}$ measures were noticed. Despite the multitude of alternatives to the gas challenges, existing literature lacks definitive conclusions regarding the best practices for the vasoactive modulation and associated analysis protocols for the TCD studies resulting in numerous metrics of cerebral 
autoregulation (Valdueza et al., 2008; Sanders et al., 2018). Regardless of methodology, an assessment of cerebrovascular autoregulation is prone to moderate noise and artifact, with low reliability and reproducibility (Lee et al., 2020). In our study, CVR was assessed using TCD which additionally suffers from a limited field of view comparing with the MRI techniques and thus it may be less reflective of the local changes in the tissue blood supply. However, due to limited access and the high cost of MRI examination, the non-invasive, bedside, and acceptable repeatable assessments using TCD, it remains still the most utilized tool to study the CBF regulation in humans. As different techniques have low correlations and target different parts of the vascular tree, they should, however, be regarded as complementary and they are recommended to be used together (Purkayastha and Sorond, 2012; Burley et al., 2021b). Another important limiting factor is that the hemodynamic effect of breath holding is lower than that of $\mathrm{CO}_{2}$ inhalation or acetazolamide injection, therefore the validity of our results should be confirmed in the future (Marcic et al., 2021). The TCD measurements are limited to the large basal arteries and can only provide an index of global rather than local CBF velocity, and it is highly operator dependent. However, all the TCD tests in our study were carried out by a single certified examiner who has 15 years of experience working with TCD, and thus we have minimized a possible interpersonal difference depending on the experience of the examiner.

The present study has, however, some important strengths. To the best of our knowledge, no data have been published on repeatedly assessed CVR measures over 24 months of observation in a well-phenotyped cohort of patients with SVD with extensive radiological markers of SVD. Ours is the first study to investigate all CVR by TCD in a wide range of participants, such as normal controls and patients with LS, VaD, and VaP. Our findings of impaired and progressive CVR alterations in the SVD subjects are important as this mechanism could contribute to exacerbating the clinical condition and may constitute a potential line of research for the treatment. A better understanding of the variations in CVR is of importance in both clinical and basic science applications (Hou et al., 2020). The majority of data on CVR are derived from the studies on individuals with asymptomatic WMLs, therefore, our study, which included homogenous groups of symptomatic SVD subjects, is important (Blair et al., 2016).

\section{REFERENCES}

Alberti, K. G., Eckel, R. H., Grundy, S. M., Zimmet, P. Z., Cleeman, J. I., Donato, K. A., et al. (2009). Harmonizing the metabolic syndrome: a joint interim statement of the International Diabetes Federation Task Force on Epidemiology and Prevention; National Heart, Lung, and Blood Institute; American Heart Association; World Heart Federation; International Atherosclerosis Society; and International Association for the Study of Obesity. Circulation 120, 1640-1645. doi: 10.1161/CIRCULATIONAHA.109.1 92644

Alwatban, M., Truemper, E. J., Al-Rethaia, A., Murman, D. L., Bashford, G. R. (2018). The breath-hold acceleration index:
In conclusion, we provided further evidence that cerebral ED occurs and progresses over time in the patients with different clinical manifestations of SVD, however, we did not observe a significant CVR difference between the subjects with SVD and radiological progression comparing with no progression group and no significant CVR alterations over time in the CG who were neurologically asymptomatic and who shared similar comorbidities to the SVD group. The longitudinal studies with larger sample sizes are needed to definite the causal conclusions and confirm these findings.

\section{DATA AVAILABILITY STATEMENT}

The raw data supporting the conclusions of this article will be made available by the authors, without undue reservation.

\section{ETHICS STATEMENT}

The studies involving human participants were reviewed and approved by Local Medical Ethics Committee, Military Institute of Medicine, Warsaw, Poland (46/WIM/2010). The patients/participants provided their written informed consent to participate in this study.

\section{AUTHOR CONTRIBUTIONS}

JS: conceptualization, methodology, validation, data curation, and project administration. JS and ES: investigation. JS and AD: writing-original draft preparation. JS and AS: supervision. All authors have read and agreed to the published version of the manuscript.

\section{FUNDING}

This study was supported by the Polish Ministry of Science and Higher Education as a research project of the Military Institute of Medicine (Warsaw, Poland, study number N N402 473840).

\section{SUPPLEMENTARY MATERIAL}

The Supplementary Material for this article can be found online at: https://www.frontiersin.org/articles/10.3389/fnagi. 2021.727832/full\#supplementary-material

a new method to evaluate cerebrovascular reactivity using transcranial Doppler. J. Neuroimaging 28, 429-435. doi: 10.1111/jon. 12508

Attwell, D., Buchan, A. M., Charpak, S., Lauritzen, M., Macvicar, B. A., and Newman, E. A. (2010). Glial and neuronal control of brain blood flow. Nature 468, 232-243. doi: 10.1038/nature09613

Blair, G. W., Doubal, F. N., Thrippleton, M. J., Marshall, I., and Wardlaw, J. M. (2016). Magnetic resonance imaging for assessment of cerebrovascular reactivity in cerebral small vessel disease: a systematic review. J. Cereb. Blood Flow Metab. 36, 833-841. doi: 10.1177/0271678X16631756

Burley, C. V., Francis, S. T., Thomas, K. N., Whittaker, A. C., Lucas, S. J. E., and Mullinger, K. J. (2021b). Contrasting measures of cerebrovascular reactivity 
between MRI and Doppler: a cross-sectional study of younger and older healthy individuals. Front. Physiol. 12:656746. doi: 10.3389/fphys.2021.656746

Burley, C. V., Francis, S. T., Whittaker, A. C., Mullinger, K. J., and Lucas, S. J. E. (2021a). Measuring resting cerebral haemodynamics using MRI arterial spin labelling and transcranial Doppler ultrasound: comparison in younger and older adults. Brain Behav. 11:e02126. doi: 10.1002/brb3.2126

Chui, H. C., Victoroff, J. I., Margolin, D., Jagust, W., Shankle, R., and Katzman, R. (1992). Criteria for the diagnosis of ischemic vascular dementia proposed by the State of California Alzheimer's disease diagnostic and treatment centers. Neurology 42, 473-480. doi: 10.1212/WNL.42.3.473

D’Andrea, A., Conte, M., Cavallaro, M., Scarafile, R., Riegler, L., Cocchia, R., et al. (2016). Transcranial Doppler ultrasonography: from methodology to major clinical applications. World J. Cardiol. 8, 383-400 doi: 10.4330/wjc.v8.i7.383

Deplanque, D., Lavallee, P. C., Labreuche, J., Gongora-Rivera, F., Jaramillo, A., Brenner, D., et al. (2013). Cerebral and extracerebral vasoreactivity in symptomatic lacunar stroke patients: a case-control study. Int. J. Stroke 8, 413-421. doi: 10.1111/j.1747-4949.2011.00755.x

Ebrahim, M. H., Khalil, S. H. M., Elbaghdady, M. M. T., and Ata Ata Shaaban, A. E. (2019). Transcranial Doppler assessment of patients with cerebral small vessel disease. J. Med. Sci. Res. 2, 75-82. doi: 10.4103/JMISR.JMISR_15_19

Fazekas, F., Chawluk, J. B., Alavi, A., Hurtig, H. I., and Zimmerman, R. A. (1987). MR signal abnormalities at $1.5 \mathrm{~T}$ in Alzheimer's dementia and normal aging. AJNR Am. J. Neuroradiol. 8, 421-426. doi: 10.2214/ajr.149.2.351

Forsberg, K. M. E., Zhang, Y., Reiners, J., Ander, M., Niedermayer, A., Fang, L., et al. (2018). Endothelial damage, vascular bagging and remodeling of the microvascular bed in human microangiopathy with deep white matter lesions. Acta Neuropathol. Commun. 6:128. doi: 10.1186/s40478-0180632-z

Fu, S., Zhang, J., Zhang, H., and Zhang, S. (2019). Predictive value of transcranial doppler ultrasound for cerebral small vessel disease in elderly patients. Arq. Neuropsiquiatr. 77, 310-314. doi: 10.1590/0004-282x20190050

Fujishima, S., Ohya, Y., Sugimori, H., Kitayama, J., Kagiyama, S., Ibayashi, S., et al. (2001). Transcranial doppler sonography and ambulatory blood pressure monitoring in patients with hypertension. Hypertens. Res. 24, 345-351. doi: 10.1291/hypres.24.345

Ghorbani, A., Ahmadi, M. J., and Shemshaki, H. (2015). The value of transcranial Doppler derived pulsatility index for diagnosing cerebral small-vessel disease. Adv. Biomed. Res. 4:54. doi: 10.4103/2277-9175.151574

Goblirsch, G., Bershow, S., Cummings, K., Hayes, R., Kokoszka, M., Lu, Y., et al. (2013). Stable Coronary Artery Disease. Vol. 5. Bloomington, MN: Institute for Clinical Systems Improvement (ICSI), 71.

Gustavsson, A. M., Stomrud, E., Abul-Kasim, K., Minthon, L., Nilsson, P. M., Hansson, O., et al. (2015). Cerebral Cerebrovasc. Dis. microbleeds and white matter hyperintensities in cognitively healthy elderly: a cross-sectional cohort study evaluating the effect of arterial stiffness. Extra 5, 41-51. doi: $10.1159 / 000377710$

Hakim, A. M. (2019). Small vessel disease. Front. Neurol. 10:1020. doi: 10.3389/fneur.2019.01020

Hou, X., Liu, P., Li, Y., Jiang, D., De Vis, J. B., Lin, Z., et al. (2020). The association between BOLD-based cerebrovascular reactivity (CVR) and end-tidal CO2 in healthy subjects. Neuroimage 207:116365. doi: 10.1016/j.neuroimage.2019.116365

Hurtig, H. I. (1993). "Vascular parkinsonism," in Parkinsonian Syndromes, eds M. B. Stern and W. C. Koller (New York, NY: Marcel Dekker), 81-83.

Inzitari, D., Pracucci, G., Poggesi, A., Carlucci, G., Barkhof, F., Chabriat, H., et al. (2009). Changes in white matter as determinant of global functional decline in older independent outpatients: three year follow-up of LADIS (leukoaraiosis and disability) study cohort. BMJ 339:b2477. doi: 10.1136/bmj.b2477

Kidwell, C. S., el-Saden, S., Livshits, Z., Martin, N. A., Glenn, T. C., and Saver, J. L. (2001). Transcranial Doppler pulsatility indices as a measure of diffuse small-vessel disease. J. Neuroimaging 11, 229-235. doi: 10.1111/j.1552-6569.2001.tb00039.x

Kim, K. W., MacFall, J. R., and Payne, M. E. (2008). Classification of white matter lesions on magnetic resonance imaging in elderly persons. Biol. Psychiatry 64, 273-280. doi: 10.1016/j.biopsych.2008.03.024

Kisler, K., Nelson, A. R., Montagne, A., and Zlokovic, B. V. (2017). Cerebral blood flow regulation and neurovascular dysfunction in Alzheimer disease. Nat. Rev. Neurosci. 18, 419-434. doi: 10.1038/nrn.2017.48
Klinzing, S., Stretti, F., Pagnamenta, A., Bèchir, M., and Brandi, G. (2021). Transcranial color-coded duplex sonography assessment of cerebrovascular reactivity to carbon dioxide: an interventional study. BMC Neurol. 21:305. doi: 10.1186/s12883-021-02310-9

Koep, J. L., Barker, A. R., Banks, R., Banger, R. R., Sansum, K. M., Weston, M. E., et al. (2020). The reliability of a breath-hold protocol to determine cerebrovascular reactivity in adolescents. J. Clin. Ultrasound 48, 544-552. doi: $10.1002 /$ jcu. 22891

Kozera, G. M., Dubaniewicz, M., Zdrojewski, T., Madej-Dmochowska, A., Mielczarek, M., Wojczal, J., et al. (2010). Cerebral vasomotor reactivity and extent of white matter lesions in middle-aged men with arterial hypertension: a pilot study. Am. J. Hypertens. 23, 1198-1203. doi: 10.1038/ajh.2010.152

Kozera, G. M., Wolnik, B., Kunicka, K. B., Szczyrba, S., Wojczal, J., Schminke, U., et al. (2009). Cerebrovascular reactivity, intima-media thickness, and nephropathy presence in patients with type 1 diabetes. Diabetes Care 32, 878-882. doi: $10.2337 / \mathrm{dc} 08-1805$

Lau, K. K., Li, L., Simoni, M., Mehta, Z., Küker, W., and Rothwell, P. M., Oxford Vascular Study (2018). Long-term premorbid blood pressure and cerebral small vessel disease burden on imaging in transient ischemic attack and ischemic stroke. Stroke 49, 2053-2060. doi: 10.1161/STROKEAHA.118.021578

Lavi, S., Gaitini, D., Milloul, V., and Jacob, G. (2006). Impaired cerebral CO2 vasoreactivity: association with endothelial dysfunction. Am. J. Physiol. Heart Circ. Physiol. 291, H1856-H1861. doi: 10.1152/ajpheart.00014.2006

Lee, Y. K., Rothwell, P. M., Payne, S. J., and Webb, A. J. S. (2020). Reliability, reproducibility and validity of dynamic cerebral autoregulation in a large cohort with transient ischaemic attack or minor stroke. Physiol. Meas. 41:095002. doi: 10.1088/1361-6579/abad49

Leoni, R. F., Mazzetto-Betti, K. C., Silva, A. C., et al. (2012). Assessing cerebrovascular reactivity in carotid steno-occlusive disease using MRI BOLD and ASL techniques. Radiol. Res. Pract. 2012:268483. doi: 10.1155/2012/268483

Longstreth, W. T. Jr., Arnold, A. M., Beauchamp, N. J. Jr., Manolio, T. A., Lefkowitz, D., Jungreis, C., et al. (2005). Incidence, manifestations, and predictors of worsening white matter on serial cranial magnetic resonance imaging in the elderly: the Cardiovascular Health Study. Stroke 36, 56-61. doi: 10.1161/01.STR.0000149625.99732.69

Maeda, H., Matsumoto, M., Handa, N., Hougaku, H., Ogawa, S., Itoh, T., et al. (1993). Reactivity of cerebral blood flow to carbon dioxide in various types of ischemic cerebrovascular disease: evaluation by the transcranial Doppler method. Stroke 24, 670-675. doi: 10.1161/01.STR.24.5.670

Marcic, M., Marcic, L.,Marcic, B., Capkun, V., and Vukojevic, K. (2021). Cerebral vasoreactivity evaluated bytranscranial color doppler and breathholding test in patients after SARS-CoV-2 infection. J. Pers. Med. 11:379. doi: 10.3390/jpm11050379

Marcos, A., Egido, J. A., Barquero, M., Fernandez, C., and de Seijas, E. V. (1997). Full range of vasodilatation tested by transcranial Doppler in the differential diagnosis of vascular and Alzheimer types of dementia. Cerebrovasc. Dis. 7, 14-18. doi: 10.1159/000108157

Markus, H., Allan, C., and Ebmeier, K. (2014). "Cerebral hemodynamics in cerebral small vessel disease," in Cerebral Small Vessel Disease, eds L. Pantoni and P. Gorelick (Cambridge: Cambridge University Press), 180-191. doi: $10.1017 / \mathrm{CBO} 9781139382694.016$

Markus, H. S., and Harrison, M. J. (1992). Estimation of cerebrovascular reactivity using transcranial Doppler, including the use of breath-holding as the vasodilatory stimulus. Stroke 23. 668-673. doi: 10.1161/01.STR.23.5.668

Markus, H. S., Lythgoe, D. J., Ostegaard, L., O'Sullivan, M., and Williams, S. C. (2000). Reduced CBF in white matter in ischemic leukoaraiosis demonstrated using quantitative exogenous contrast based perfusion MRI. J. Neurol. Neurosurg. Psychiatry 69, 48-53. doi: 10.1136/jnnp.69.1.48

McDonnell, M. N., Berry, N. M., Cutting, M. A., Keage, H. A., Buckley, J. D., and Howe, P. R. (2013). Transcranial Doppler ultrasound to assess cerebrovascular reactivity: reliability, reproducibility and effect of posture. PeerJ 1:e65. doi: 10.7717/peerj.65

Molina, C., Sabin, J. A., Montaner, J., Rovira, A., Abilleira, S., and Codina, A. (1999). Impaired cerebrovascular reactivity as a risk marker for first-ever lacunar infarction: a case-control study. Stroke 30, 2296-2301. doi: 10.1161/01.STR.30.11.2296

Muoio, V., Persson, P. B., and Sendeski, M. M. (2014). The neurovascular unit concept review. Acta Physiol. 210, 790-798. doi: 10.1111/apha.12250 
Nam, K. W., Kwon, H. M., and Lee, Y. S. (2020). Distinct association between cerebral arterial pulsatility and subtypes of cerebral small vessel disease. PLoS ONE 15:e0236049. doi: 10.1371/journal.pone.0236049

Ojeda, A. E., Martinez, H. R., Rivera, F. G., Garza, J. M. E., and Medina, H. C. (2017). Cerebral vasoreactivity in Parkinson's Disease: a cross-sectional pilot study in a hispanic cohort. J. Alzheimers. Dis. Parkinsonism 7:336. doi: 10.4172/2161-0460.1000336

Pánczél, G., Bönöczk, P., and Nagy, Z. (2002). A vazoreaktivitás zavara agytörzsi és hemisphaerialis kisérbetegségben: összehasonlító vizsgálat [Impairment of vasoreactivity in brainstem and hemispheral small vessel disease: comparative study]. Ideggyogy Sz 55, 95-101.

Panerai, R. B. (2009). Transcranial Doppler for evaluation of cerebral autoregulation. Clin. Auton. Res. 19, 197-211. doi: 10.1007/s10286-009-0011-8

Panerai, R. B., Deverson, S. T., Mahony, P., Hayes, P., and Evans, D. H. (1999). Effects of $\mathrm{CO} 2$ on dynamic cerebral autoregulation measurement. Physiol. Meas. 20, 265-275. doi: 10.1088/0967-3334/20/3/304

Pantoni, L. (2010). Cerebral small vessel disease: from pathogenesis and clinical characteristics to therapeutic challenges. Lancet Neurol. 9, 689-701. doi: 10.1016/S1474-4422(10)70104-6

Prins, N. D., van Straaten, E. C., van Dijk, E. J., Simoni, M., van Schijndel, R. A., Vrooman, H. A., et al. (2004). Measuring progression of cerebral white matter lesions on MRI: visual rating and volumetrics. Neurology 62, 1533-1539. doi: 10.1212/01.WNL.0000123264.40498.B6

Purkayastha, S., and Sorond, F. (2012). Transcranial Doppler ultrasound: technique and application. Semin. Neurol. 32, 411-420. doi: 10.1055/s-0032-1331812

Ringelstein, E. B., Sievers, C., Ecker, S., Schneider, P. A., and Otis, S. M. (1988). Noninvasive assessment of $\mathrm{CO} 2$-induced cerebral vasomotor response in normal individuals and patients with internal carotid artery occlusions. Stroke 19, 963-969. doi: 10.1161/01.STR.19.8.963

Sam, K., Crawley, A. P., Conklin, J., Poublanc, J., Sobczyk, O., Mandell, D. M., et al. (2016). Development of white matter hyperintensity is preceded by reduced cerebrovascular reactivity. Ann. Neurol. 80, 277-285. doi: 10.1002/ana.24712

Sanders, M. L., Claassen, J. A. H. R., Aries, M., Bor-Seng-Shu, E., Caicedo, A., Chacon, M., et al. (2018). Reproducibility of dynamic cerebral autoregulation parameters: a multi-centre, multi-method study. Physiol. Meas. 39:125002. doi: 10.1088/1361-6579/aae9fd

Schulc, E., Pallauf, M., Mueller, G., Wildbahner, T., and Them, C. (2015). Is the barthel index an adequate assessment tool for identifying a risk group in elderly people living at home? Int. J. Nurs. Clin. Pract. 2:140. doi: 10.15344/2394-4978/2015/140

Settakis, G., Lengyel, A., Molnár, C., Bereczki, D., Csiba, L., and Fülesdi, B. (2002). Transcranial Doppler study of the cerebral hemodynamic changes during breath-holding and hyperventilation tests. J. Neuroimaging 12, 252-258. doi: 10.1111/j.1552-6569.2002.tb00129.x

Shim, Y., Yoon, B., Shim, D. S., Kim, W., An, J.-Y., and Yang, D.-W. (2015). Cognitive correlates of cerebral vasoreactivity on transcranial doppler in older adults. J. Stroke Cerebrovasc. Dis. 24, 1262-1269. doi: 10.1016/j.jstrokecerebrovasdis.2015.01.031

Silvestrini, M., Vernieri, F., Pasqualetti, P., Matteis, M., Passarelli, F., Troisi, E., et al. (2000). Impaired cerebral vasoreactivity and risk of stroke in patients with asymptomatic carotid artery stenosis. JAMA 283, 2122-2127. doi: 10.1001/jama.283.16.2122

Sleight, E., Stringer, M. S., Marshall, I., Wardlaw, J. M., and Thrippleton, M. J. (2021). Cerebrovascular reactivity measurement using magnetic resonance imaging: a systematic review. Front. Physiol. 12:643468. doi: 10.3389/fphys.2021.643468

Soman, S., Dai, W., Dong, L., Hitchner, E., Lee, K., Baughman, B. D., et al. (2020). Identifying cardiovascular risk factors that impact cerebrovascular reactivity: an ASL MRI study. J. Magn. Reson. Imaging 51, 734-747. doi: 10.1002/jmri.26862

Staals, J., Makin, S. D., Doubal, F. N., Dennis, M. S., and Wardlaw, J. M. (2014). Stroke subtype, vascular risk factors, and total MRI brain small-vessel disease burden. Neurology 83, 1228-1234. doi: 10.1212/WNL.0000000000000837

Staszewski, J., Piusińska-Macoch, R., Skrobowska, E., Brodacki, B., Pawlik, R., Dutkiewicz, T., et al. (2013). Significance of haemodynamic and haemostatic factors in the course of different manifestations of cerebral small vessel disease: the SHEF-CSVD Study-study rationale and protocol. Neurosci. J. 2013:424695. doi: 10.1155/2013/424695

Staszewski, J., Skrobowska, E., Piusińska-Macoch, R., Brodacki, B., and Stepień, A. (2019). Cerebral and extracerebral vasoreactivity in patients with different clinical manifestations of cerebral small-vessel disease: data from the significance of hemodynamic and hemostatic factors in the course of different manifestations of cerebral small-vessel disease study. J. Ultrasound Med. 38, 975-987. doi: 10.1002/jum.14782

Stevenson, S. F., Doubal, F. N., Shuler, K., and Wardlaw, J. M. (2010). A systematic review of dynamic cerebral and peripheral endothelial function in lacunar stroke versus controls. Stroke 41, e434-e442. doi: 10.1161/STROKEAHA.109.569855

Ten Dam, V. H., van den Heuvel, D. M., de Craen, A. J., Bollen, E. L., Murray, H. M., Westendorp, R. G., et al. (2007). Decline in total cerebral blood flow is linked with increase in periventricular but not deep white matter hyperintensities. Radiology. 243, 198-203. doi: 10.1148/radiol.2431052111

Terborg, C., Gora, F., Weiller, C., and Röther, J. (2000). Reduced vasomotor reactivity in cerebral microangiopathy: a study with near-infrared spectroscopy and transcranial Doppler sonography. Stroke 31, 924-929. doi: 10.1161/01.STR.31.4.924

Thrippleton, M. J., Shi, Y., Blair, G., Hamilton, I., Waiter, G., Schwarzbauer, C., et al. (2018). Cerebrovascular reactivity measurement in cerebral small vessel disease: rationale and reproducibility of a protocol for MRI acquisition and image processing. Int. J. Stroke 13, 195-206. doi: 10.1177/1747493017730740

Tsivgoulis, G., and Alexandrov, A. V. (2008). Cerebral hemodynamics in acute stroke: pathophysiology and clinical implications. J. Vasc. Interv. Neurol. 1, 65-69.

Tzourio, C., Levy, C., Dufouil, C., Touboul, P. J., Ducimetière, P., Alpérovitch, A., et al. (2001). Low cerebrl blood flow velocity and risk of white matter hyperintensities. Ann. Neurol. 49, 411-414. doi: 10.1002/ana.82

Urbanova, B. S., Schwabova, J. P., Magerova, H., Jansky, P., Markova, H., Vyhnalek, M., et al. (2018). Reduced cerebrovascular reserve capacity as a biomarker of microangiopathy in Alzheimer's disease and mild cognitive impairment. J. Alzheimer's Dis. 63, 465-477. doi: 10.3233/JAD-170815

Valdueza, J. M., Schreiber, S. J., Roehl, J.-E., and Klingebiel, R. (2008). “Intracranial hemodynamics and functional tests," in Neurosonology and Neuroimaging of Stroke, ed J. M. Valdueza (New York, NY: Thieme), 79-88.

Wardlaw J. M., Smith E. E., Biessels G. J., Cordonnier, C., Fazekas, F., Frayne, R., et al. (2013). Neuroimaging standards for research into small vessel disease and its contribution to ageing and neurodegeneration. Lancet Neurol. 12, 822-838. doi: 10.1016/S1474-4422(13)70124-8

Zijlmans, J. C. M., Daniel, S. E., Hughes, A. J., Révész, T., and Lees, A. J. (2004). Clinicopathological investigation of vascular parkinsonism, including clinical criteria for diagnosis. Mov. Disord. 19, 630-640. doi: 10.1002/mds.20083

Zlokovic, B. V. (2011). Neurovascular pathways to neurodegeneration in Alzheimer's disease and other disorders. Nat. Rev. Neurosci. 12, 723-738. doi: $10.1038 / \mathrm{nrn} 3114$

Conflict of Interest: The authors declare that the research was conducted in the absence of any commercial or financial relationships that could be construed as a potential conflict of interest.

Publisher's Note: All claims expressed in this article are solely those of the authors and do not necessarily represent those of their affiliated organizations, or those of the publisher, the editors and the reviewers. Any product that may be evaluated in this article, or claim that may be made by its manufacturer, is not guaranteed or endorsed by the publisher.

Copyright (C) 2021 Staszewski, Dębiec, Skrobowska and Stępień. This is an open-access article distributed under the terms of the Creative Commons Attribution License (CC BY). The use, distribution or reproduction in other forums is permitted, provided the original author(s) and the copyright owner(s) are credited and that the original publication in this journal is cited, in accordance with accepted academic practice. No use, distribution or reproduction is permitted which does not comply with these terms. 